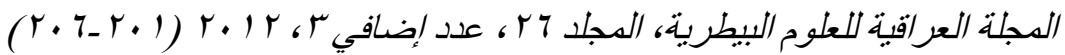

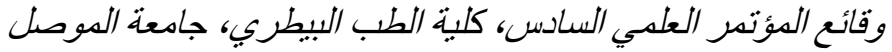 \\ تواجد أنواع الإيميريا في فروج اللحم في منطقة الحمدانية ـ نينوى \\ مصطفى سالم شاكر النعمة و أحلام فتحي الطائي \\ فرع الأحياء المجهرية، كلية الطب البيطري، جامعة الموصل، الموصل، العراق
}

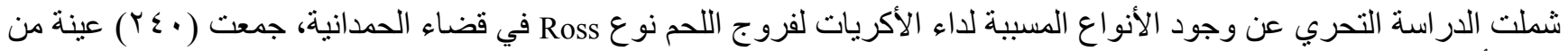

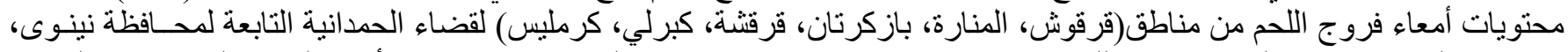

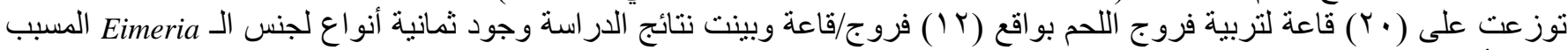
لداء الأكريات في فروج اللحم (E.necatrix, E.maxima, E.mivati, E.mitis, E.brunetti, E.acervulina, E.praecox, Eimeria tenella) بلغت النسبة الكلية للأصابة بالأكريات في فروج اللحم

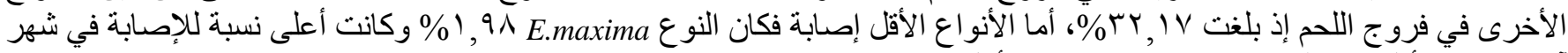

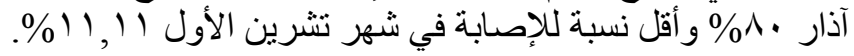

\section{Incidence of Eimeria spp in broilers in Al-Hamdania region-Ninevah}

\section{M.S.S. ALNeema and A.F. Al-Taee}

Department of Microbiology, College of Veterinary Medicine, Univercity of Mosul, Mosul ,Iraq

\begin{abstract}
This study was conducted to investigate the incidence and intensity of coccidial species in broilers (type Ross) in AlHamdania region, two hundred and forty samples were collected from the intestinal content of the broilers obtained from (20) farms located in the suburban regions: (Karakosh, Manara, Bazgertan, Karkasha, Kaberly,Karamles) within Al-Hamdania region by obtaining (12) birds from each farms. The results revealed the prescience of eight of Eimeria species in broilers these species were: Eimeria tenella; E.necatrix; E.maxima; E.mivati; E.mitis; E.brunetti; E.acervulina; E.praecox. The total percentage of broiler coccidiosis was $45 \%$, the percentage of infection variant in different months, the highest, $80 \%$ during March and the lowest during October $11.11 \%$, Eimeria tenella had the highest percentage of isolation in broilers $32.17 \%$, while the lowest percentage of infection were E.maxima1.98\%inbroilers.
\end{abstract}

Available online at http://www.vetmedmosul.org/ijvs

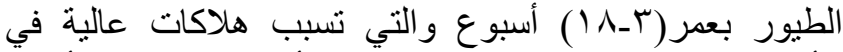
المقدمة

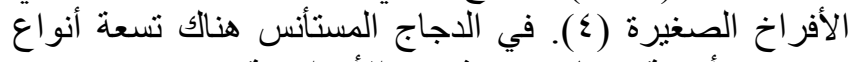

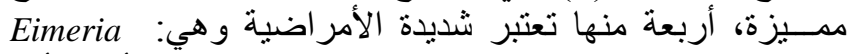
bruneti, E.maxima, E.necatrix and E.tenella امر اضية وهي: E.acervulina, E.mitis and E.mivati و الأنير النوعان

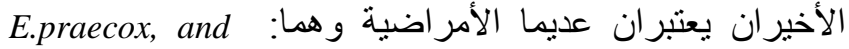

(0) E.hagani

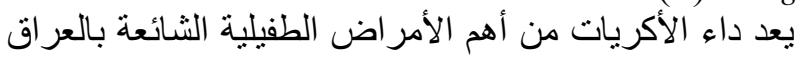

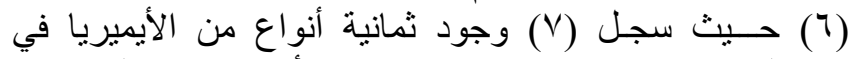

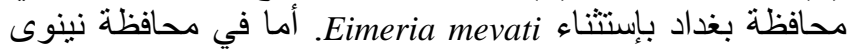

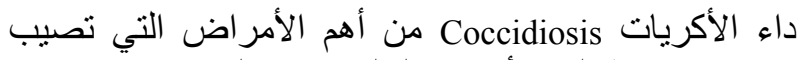

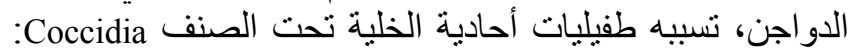
Eimeriorina Subclass

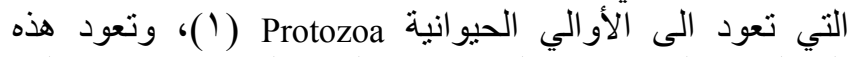

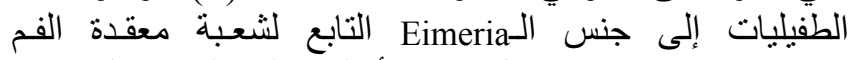
Apicomplexa

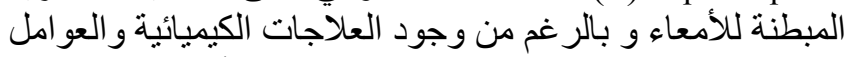

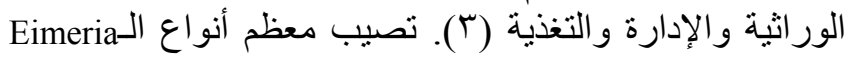




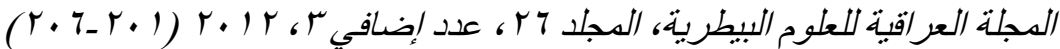

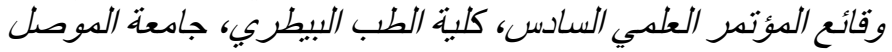

أجريت الفحوصات المختبرية في مختبر البحوث الطفيلية

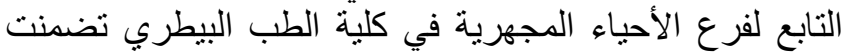

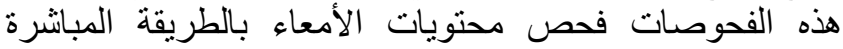

حسب طريقة (9) و إستخدام تقنية الطفو حسب طريقة (· • (1).

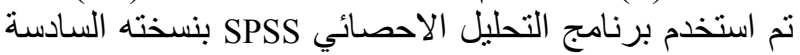

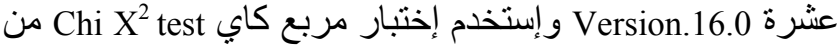
أجل تحديد وجود فروقات معنوية بين المجاميع المختلفة عندارئ

(1) (1)

النتائج

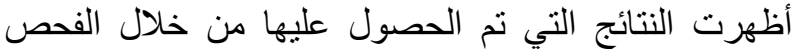

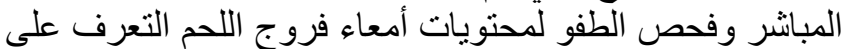

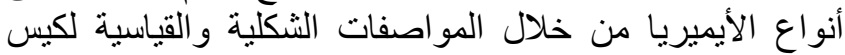

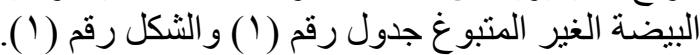

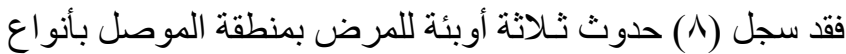
E.necatrix و Eimeria tenella

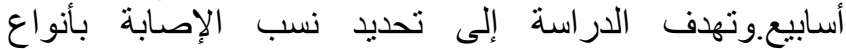

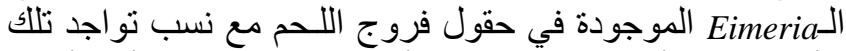

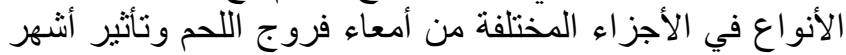

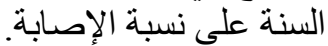

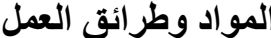

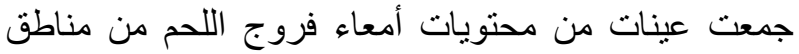
(قرة قوش، المنارة، بازركتان، قرقثة، كبرلي، كرم ليس) التابعة

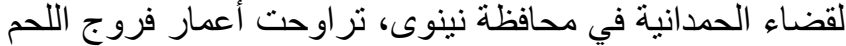

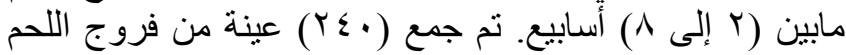

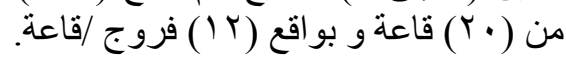

جدول ( ) : أنو اع الإيميريا المشخصة في فروج اللحم حسب المواصفات القياسيةو الثكلية لكيس البيضة الغير المتبوغ.

\begin{tabular}{|c|c|c|c|c|c|}
\hline \multicolumn{6}{|c|}{ المو اصفات الثكلية لكيس البيضة الغير المتبوغ } \\
\hline الثكل & القبعة & 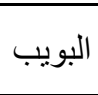 & 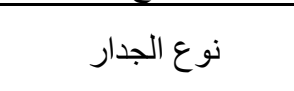 & متوسط الطول × العرض (بالمايكرون) & نوع الإيميريا المشخصة \\
\hline & & & & $1 \wedge, \wedge T \times Y r, q \wedge$ & \\
\hline بيضوي & - & - & 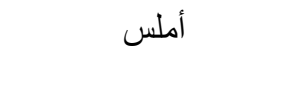 & 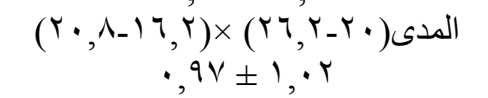 & Eimeria tenella \\
\hline 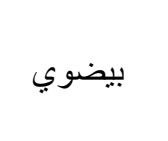 & - & - & أملس و عديم اللون & 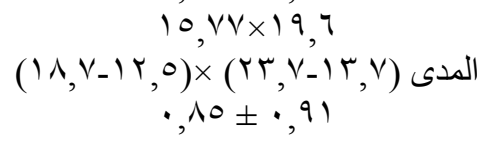 & Eimeria necatrix \\
\hline بيضوي & - & - & أملس ورقيق من النهاية & 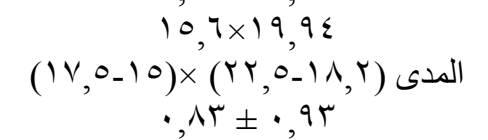 & Eimeria acervulina \\
\hline بيضوي كبير & - & - & خشن ولونه أصفر & 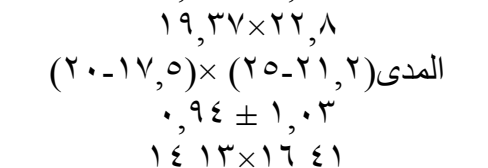 & Eimeria maxima \\
\hline 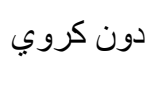 & - & - & 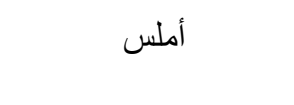 & 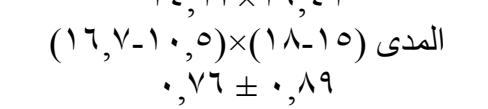 & Eimeria mitis \\
\hline إهليلجي إلى & & & & $i r, v 1 \times i 0$ & \\
\hline عبضوي & - & + & أملس و عديم اللون & 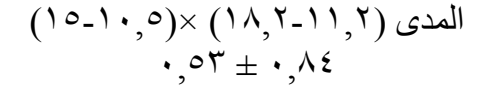 & Eimeria mivati \\
\hline & & & & $19,1 \times r r, \wedge \varepsilon$ & \\
\hline 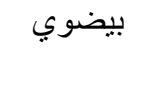 & - & - & أملس & 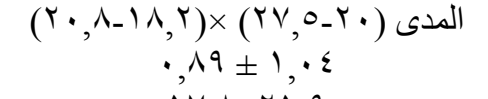 & Eimeria brunetti \\
\hline 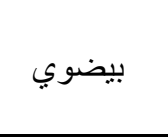 & - & - & أملس و عديم اللون & 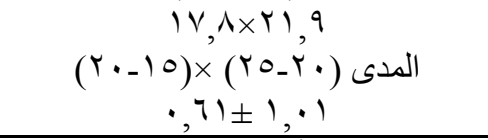 & Eimeria praecox \\
\hline
\end{tabular}




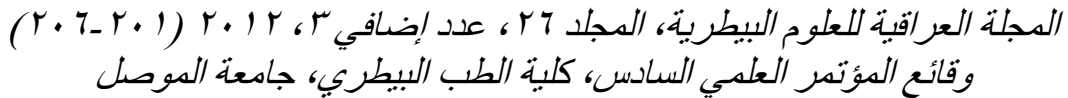

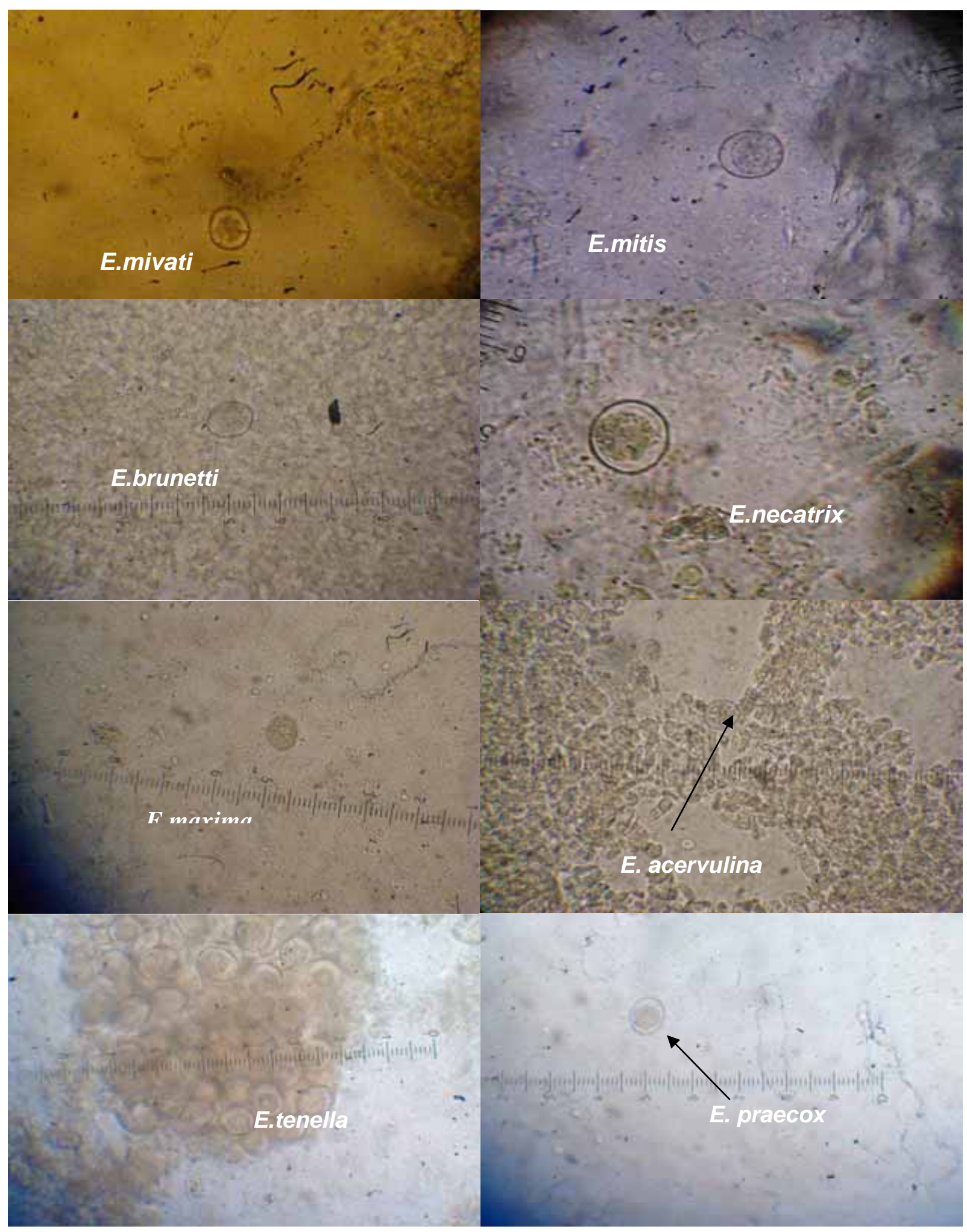

شكل رقم ( (): أكياس البيض الغير المتبوغة للأنواع الثمانية المشخصة للجنس Eimeria في فروج اللحم قوة التكبير ـ. 


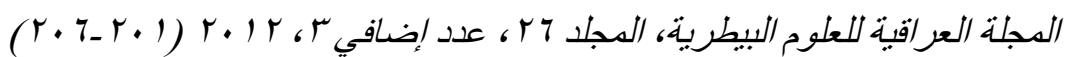

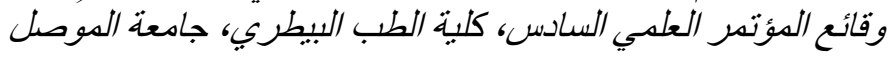

أظهرت نتائج الفحص المجهري لمحتويات الأمعاء ظهور

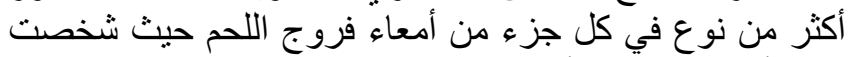
في الأثني عشري الأنواع (E.acervulina,E.praecox,E.mivati)

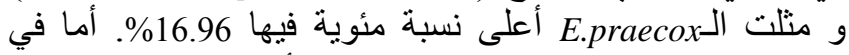

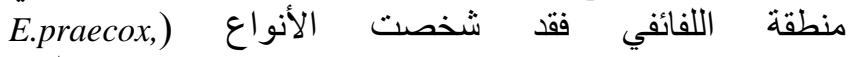
(E.brunetti,E.acervulina,E.maxima,E.mivati,E.mitis نسبة الإصابةبال، E.mivati أعلى نسبة مئوية فيها E8.03\%. أما في E. Eي E.brunetti,) منطقة الصائم E.necatrix,E.mivati,E.mitis مئوية فيها 22.32\%، أما في منطقة الأعورين فكانت الأنواع اعلى

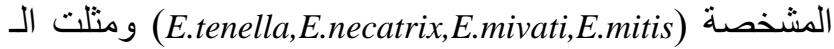
E.tenella

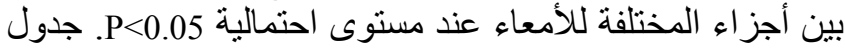
رقم (T). بلغت النسبة المئوية للإصابة بالإيميريا في فروج اللحم 0؛

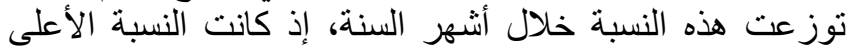

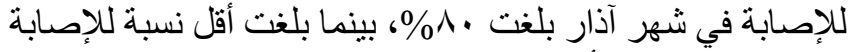

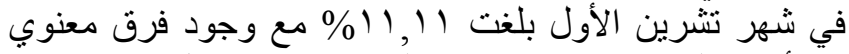

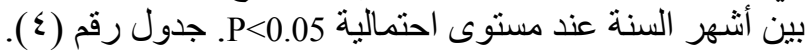

تم تشخيص ثمانبة أنواع في فروج اللحم وهي (Eimeria tenella, E.mivati, E.mitis, E.brunetti, E.acervulina, , (E.necatrix, E.maxima, E.praecox , \%5.44, \%11.88, \%1.98 , \% 11.38 \%16.83, \%32.17

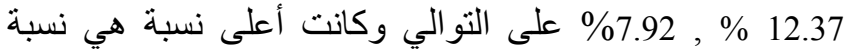

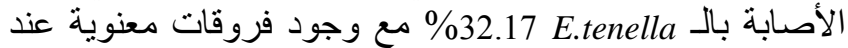

مستوى إحتمالية P>0.05 جدول رقم (r).

جدول (Y): نسب الأنواع المشخصة من الإيميريا في حقول

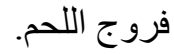

\begin{tabular}{|c|c|c|}
\hline لكبة نوع المئوية & أنواع الإيميريا & نوع الدجاج \\
\hline$b \vee .9 r$ & Eimeria mitis & \multirow{8}{*}{ فروج اللحم } \\
\hline d $17 . \Lambda \mu$ & E.mivati & \\
\hline$c 11 . \wedge \Lambda$ & E.necatrix & \\
\hline$b 0 . \leqslant \varepsilon$ & E.acervulina & \\
\hline c $11 . r$ & E.praecox & \\
\hline c IT.rV & E.brunutti & \\
\hline a 1.91 & E.maxima & \\
\hline $\mathrm{e}^{\mathrm{Tr}} . \mathrm{IV}$ & E.tenella & \\
\hline
\end{tabular}

جدول (r): النسبة المئوية لوجود أنواع الإيميريا المشخصة حسب الموقع في أمعاء فروج اللح.

\begin{tabular}{|c|c|c|c|c|c|}
\hline النسبة المئوية & 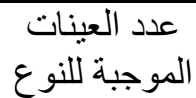 & من العدد الكينات للعينات الموجبة & أنواع الإيميريا المشخصة & & الألجزءء المفحوص من \\
\hline 6.25 & 7 & \multirow{3}{*}{ - } & Eimeria acervulina & \multirow{3}{*}{$\mathrm{a}$} & \multirow{9}{*}{ اللفائفي } \\
\hline 16.96 & 19 & & Eimeria praecox & & \\
\hline 10.71 & 12 & & Eimeria mivati & & \\
\hline 7.14 & 8 & \multirow{6}{*}{112} & Eimeria praecox & \multirow{6}{*}{$\mathrm{a}$} & \\
\hline 1.78 & 2 & & Eimeria brunetti & & \\
\hline 2.67 & 3 & & Eimeria acervulina & & \\
\hline 3.57 & 4 & & Eimeria maxima & & \\
\hline 8.03 & 9 & & Eimeria mivati & & \\
\hline 6.25 & 7 & & Eimeria mitis & & \\
\hline 22.32 & 25 & \multirow{6}{*}{112} & Eimeria brunetti & & \\
\hline 5.35 & 6 & & Eimeria necatrix & \multirow{5}{*}{$\mathrm{b}$} & الصائم \\
\hline 6.25 & 7 & & Eimeria mivati & & jejunum \\
\hline 7.14 & 8 & & Eimeria mitis & & \\
\hline 84.82 & 95 & & Eimeria tenella & & \multirow{4}{*}{$\begin{array}{r}\text { الأعورين } \\
\text { caecum }\end{array}$} \\
\hline 8.03 & 9 & & Eimeria necatrix & & \\
\hline 6.25 & 7 & \multirow{2}{*}{112} & Eimeria mivati & \multirow[t]{2}{*}{$\mathrm{c}$} & \\
\hline 3.57 & 4 & & Eimeria mitis & & \\
\hline
\end{tabular}

الأحرف الصغيرة المختلفة عموديا تشير إلى وجود فروقات معنوية عند مستوى إحتماليةP>0.05. 


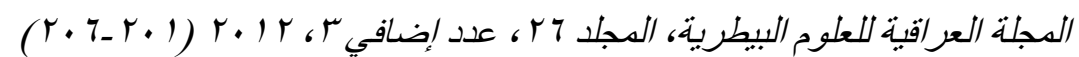

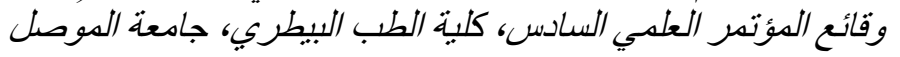

جدول (ع): النسبة المئوية للأصابة بالإيميريا لفروج اللحم خلال أشهر السنة.

\begin{tabular}{|c|c|c|c|c|}
\hline للأصبابة المئوية & عدد العينات & عدد العينات & عدد القاعات & أشنهر السنة \\
\hline a 11,11 & $\varepsilon$ & ry & $r$ & تشرين الأول 9 . . r \\
\hline e 77,77 & $\wedge$ & ir & 1 & تشرين الثاني \\
\hline$b r \cdot, 90$ & rq & $\wedge \varepsilon$ & V & كانون الأول \\
\hline c $\leqslant 1,77$ & 1. & $r \varepsilon$ & r & كانون الثاني • · · ب \\
\hline 0. & ir & $r \leq$ & r & شباط \\
\hline $\mathrm{f} \quad \wedge$. & $\varepsilon \wedge$ & 7. & 0 & آذار \\
\hline$\leqslant 0$ & $1 \cdot 1$ & $r \leqslant$. & $r \cdot$ & المجموع \\
\hline
\end{tabular}

الأحرف الصغيرة المختلفة عموديا تشير إلى وجود فروقات معنوية عند مستوى إحتمالية P>0.05.

$6 \% 0, \Sigma \varepsilon \quad$ E.acervulina $\quad \% \%$, $9 \mathrm{r} \quad$ E.mitis $\% 11, \Gamma \wedge$

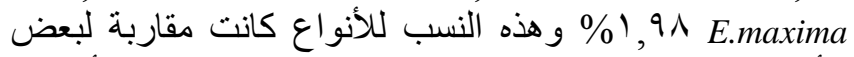

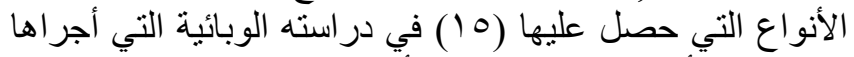

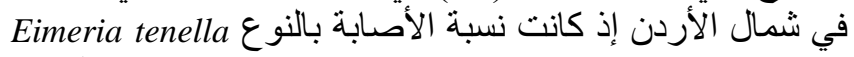

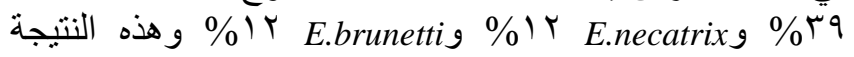
تعزى للتشابه في الظروف البيئية في تلإك المنطقة مع الظروف

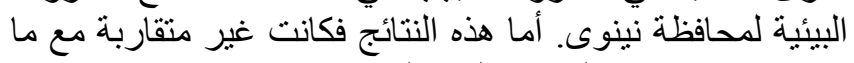

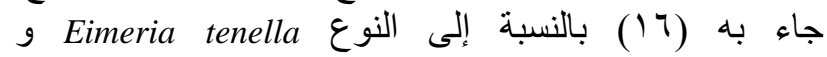

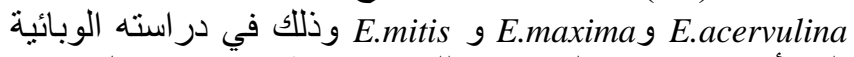

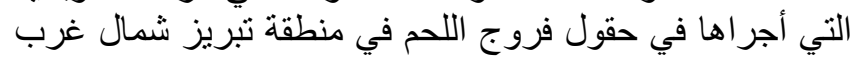

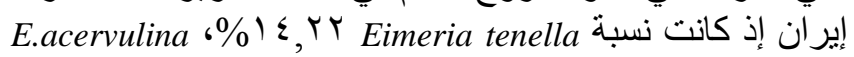

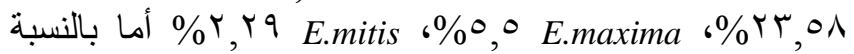

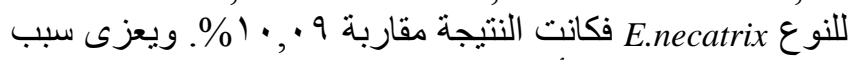

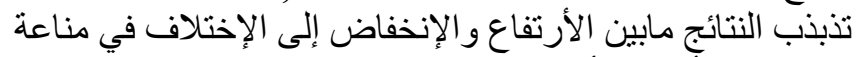
الطائر ضد أنواع الأنين الأيميريا (1) (1).

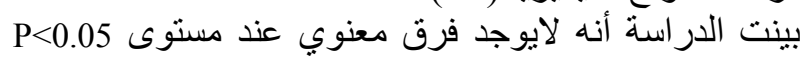

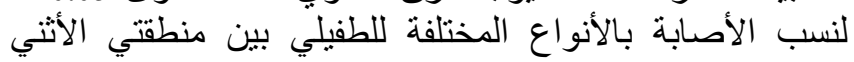

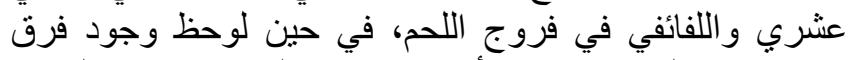

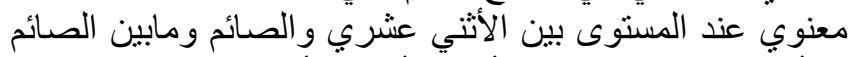

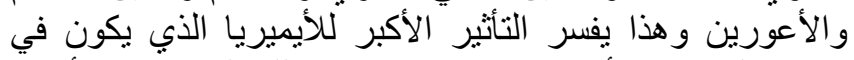

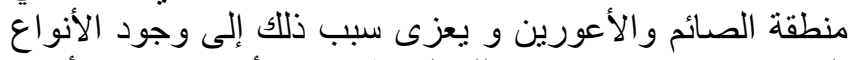

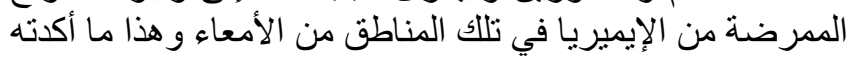

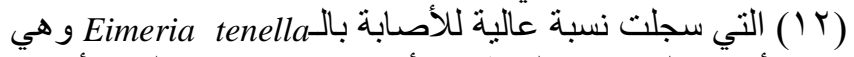

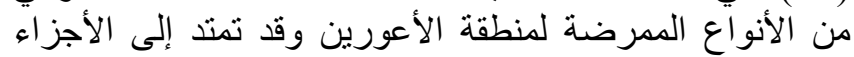

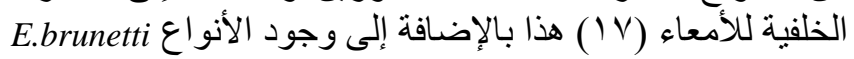

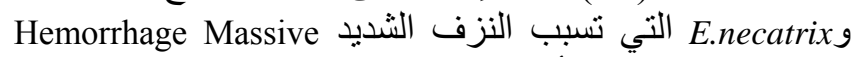
في تلأك المناطق من الأمعاء (1) (1). وأظهرت النتائج للأصابة بالأنكريات الأنات أن النسبة الأعلى

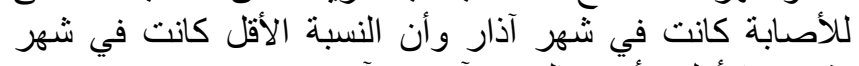

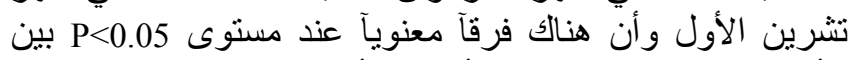

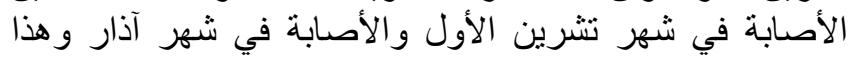

المناقشة

أظهرت نتائج الدراسة أن النسبة المئوية للعينات الموجبة من المن المبات

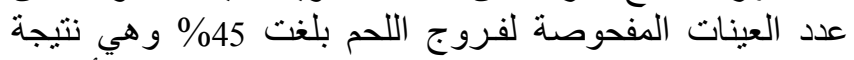
مقاربة لما جاءت به اللـ(Y I) في در استها المسحية التي أجرتها

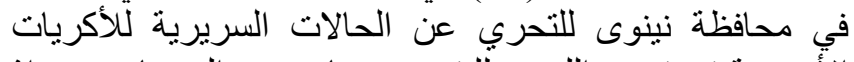

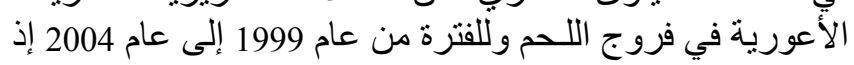

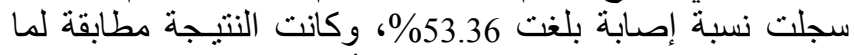

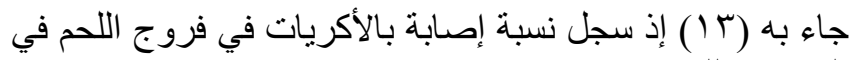

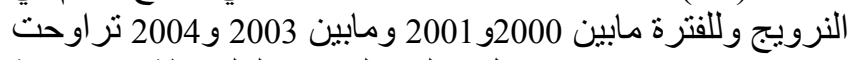

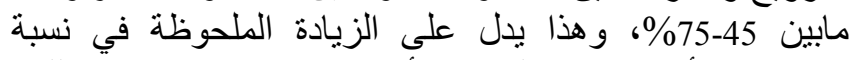

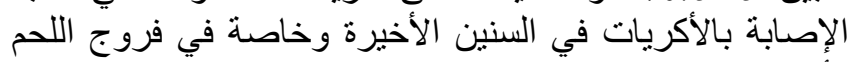

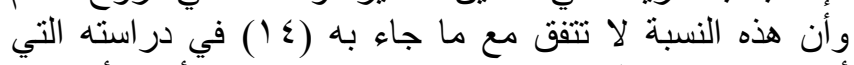

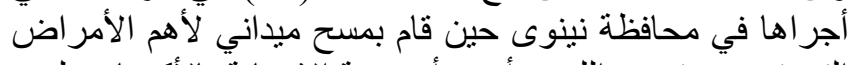

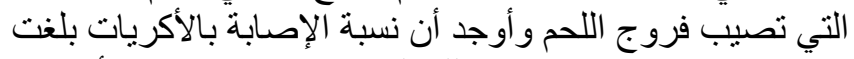

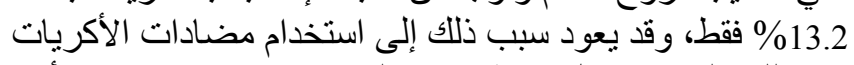

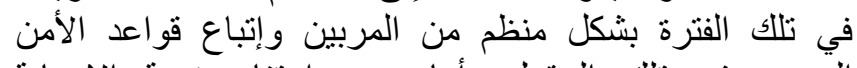

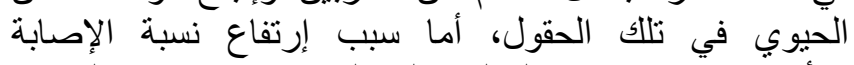

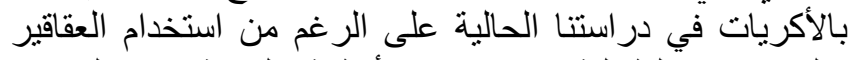

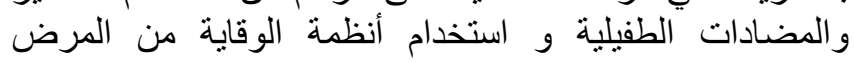

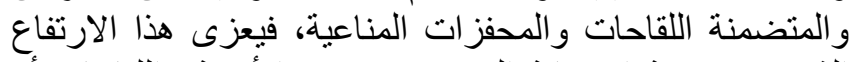

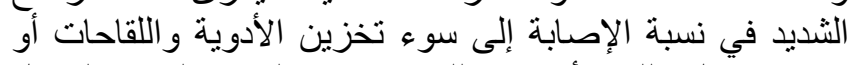

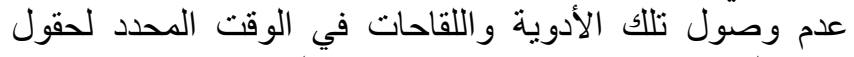

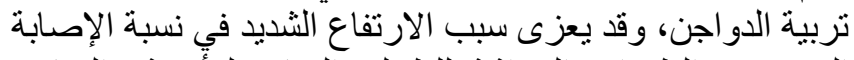

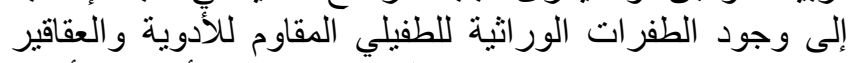

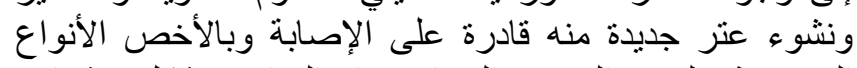

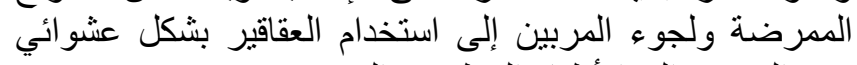
دون الرجوع إلى الأطباء البيطريين المبن المختصين.

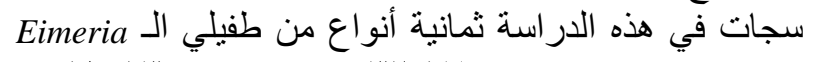

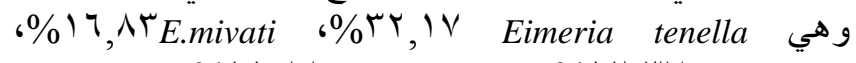

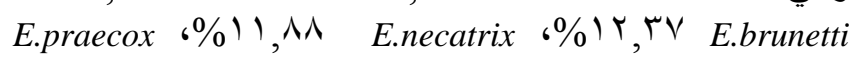




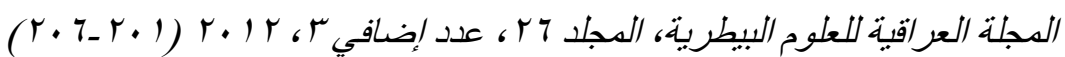

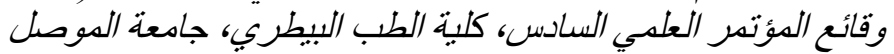

out breaks in New Hamshire and broiler type chickens at Mosul.Faculty of Agriculture and Veterinary Medicine.University of Mosul. Mosul.Iraq. 1971.

9. Foreyt WJ. Veterinary Parasitology. $5^{\text {th }}$ edition.Low state university press (U.S.A.). $2001 ; 5-7$.

10. Urquhart GM, Armour J, Duncan JL, Dunn A M , Jennings FW.. Veterinary Parasitology. ${ }^{\text {rd }}$ ed. Blackwell science ltd.. 2001; 223-231.

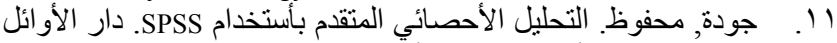

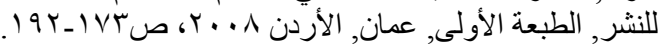

12. Al-Taee AFM.Incidence of broiler caecal coccidiosis in Ninevah Governorate 1999-2004.Iraqi J. of Vete.Scie.. 2007; 21(1):45-63.

13. Haug A, Gjevre AG, Thebo P, Mattsson JG, Kaldhusdal,M. Coccidial infections in commercial broilers: epidemiological aspects and comparison of Eimeria species identification by morphometric and polymerase chain reaction techniques.Avian Pathology. 2008;37; 2:161-170.

ء ا. العطار, مز احم ياسين. مسح ميداني لأمر اض فروج اللحم في محافظة

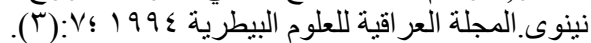

15. Al-Natour MQ, Suleiman MM., Abo-Shehada MN.Flock-level prevalence of Eimeria species among broiler chicks in northern Jordan.Preve. Vet. Med. 2002;53(3):305-310.

16. Nematollahi A, Moghaddam Gh, Pourabad RF. Prevalence of Eimeria species among broiler chicks in Tabriz (Northwestern of Iran).Munis Entomo. and Zool. 2009;4(1):53-58.

17. Saif Y M, Barnes HJ, Glisson JR, Fadly A M, McDouglald L R , Swayne D E. Diseased of Poultry, $11^{\text {th }}$,Iowa state press. (U.S.A)2003;974-985.

18. Jordan F, Pattison M, Alexander D, McMulin , Bradbury. Poultry Diseases. $6^{\text {th }}$ ed. Saunders Elsevier. Philadelphia. U.S.A. 2008;444451.

19. Etuk E.B, Okoli IC, Uko MU. Prevalence and management issues associated with poultry coccidiosis in Abak Agricultural Zona of Akwa Ibom State,Nigeria. Intern. Jour. of Poult. Scie. 2004;3(2):135-139.

20. Alawa CBI, Mohammed AK, Oni OO, Adeyinka IA, Lamidi OS , Adam AM. Prevalence and seasonality of common health problems in Sokoto Gudali cattle at a beef research station in the Sudan ecological zone of Nigeria.Nig.J.Anim.Prod. 2001;28:224-228.

21. Halle PD, Umoh JU, Saidu L, Abdu PA. Disease of poultry in Zaria, Nigeria: A ten-year analysis of clinical records. Nig.J. Anim.Prod. 1998;25:88-92.

22. Oluyemi JA , Roberts FA. Poultry production in warm wet climate.. Low cost edition. Macmillan publishers Ltd. London.. 1979.:141-147.

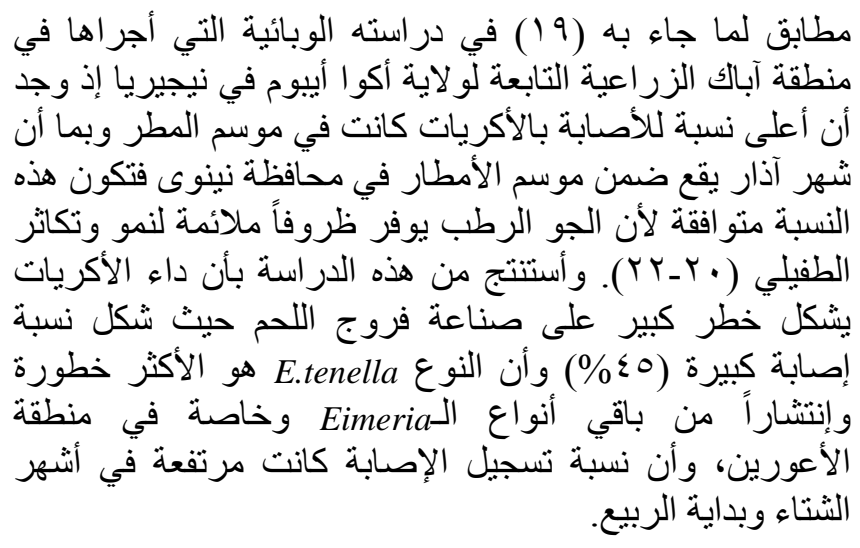

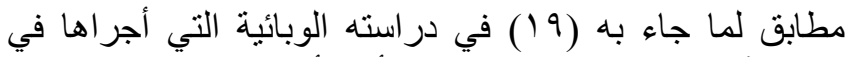

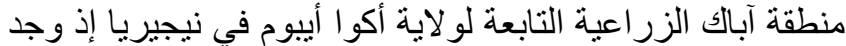

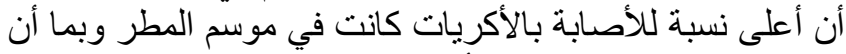

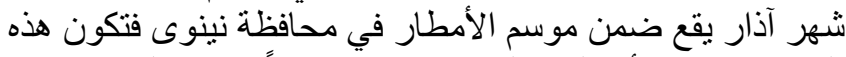

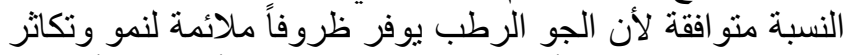

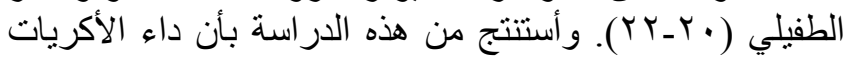

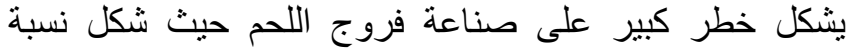

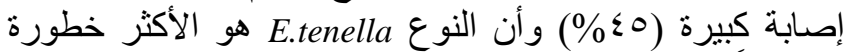

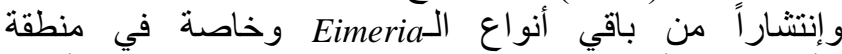
الأعورين، وأن نسبة تسجيل الإصنابة كانت مرتفعة في أثنهر فئرة فئرة الثتاء وبداية الربيع.

الثكر و التقدير

يتقدم الباحثان بالثكر و التقدير إلى عمادة كلية الطب البيطري لمساهمتها الفاعلة في إتمام هذا البحث.

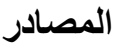

1. Bowman DD, Lynn RC, Eberhard ML. Parasitology for veternarians. $8^{\text {th }}$ ed. U.S. A. $2003 ; 251-269$.

2. Shirley MW. Eimeria species and strains of chickens. Guidelines on Techniques in Coccidiosis Research. European commission, Directorate General XII, Science Research and Development, Agriculture Biotechnology, L-2820 Luxemburge,. 1995 ;1-34.

3. Magner BRI. Anticoccidials.Veterinary applied pharmacology and therapeutics. $5^{\text {th }}$ ed.ELBS, Bailliere Tindall,London. 1991; 549-563.

4. McDougald LF , Mattiello RA. A survey of coccidia on 43 poultry farms in Argentina.Avian Dis. 1997;41(3):923-929.

5. Thebo P, Uggla A, Hooshmand-Rad P. Identification of seven Eimeria species in Swedish domestic fowl. Avian Pathol. 1998; 27:613-617.

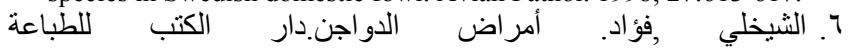

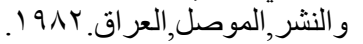

7. Latif BM, Ali $S$, Versenyi I. Chicken coccidiosis in Iraq.Acta veteran area. 1974; 24(3):132-135.

8. Abu-El-azm IM, Nadim MAC.Recognition of coccidial species Eimeria tenella and Eimeria necatrix as the etiological factor in three 